Mawaddah Azman, MD

Balwant Singh Gendeh, MBBS, MS (ORLHNS)'

Siti Aishah Mat Ali, MBBCh, DCP²

'Department of Otorhinolaryngology

Head and Neck Surgery

Faculty of Medicine, Universiti Kebangsaan Malaysia

Kuala Lumpur, Malaysia

${ }^{2}$ Department of Pathology

Faculty of Medicine, Universiti Kebangsaan Malaysia

Kuala Lumpur, Malaysia

\title{
Extramedullary Plasmacytoma of the Nasopharynx: A Rare Tumour with 7-Year Follow Up
}

\begin{abstract}
Objective: To report a case of extramedullary plasmacytoma, a rare localized tumour involving the head and neck region in a 56-year -old gentleman.
\end{abstract}

\section{Methods:}

Design: Case Report

Setting: Tertiary University Referral Center

Patient: One

Result: The patient presented with a 5-month history of right-sided nasal obstruction and intermittent epistaxis in 2003. Nasal endoscopy revealed a friable, dark red mass arising from the roof of the nasopharynx, occluding the right choana. No invasion of adjacent tissues or cervical lymphadenopathy was evident. A biopsy of the mass was diagnosed as plasmacytoma. Serum and urine electrophoresis failed to detect any monoclonal bands. All other screening tests to rule out multiple myeloma were negative. These findings confirmed the diagnosis of extramedullary plasmacytoma. He recieved radiotherapy to the nasopharynx of $50 \mathrm{~Gy}$ for a total of 23 fractions. No recurrence was noted at 7-year follow-up.

Conclusion: Extramedullary plasmacytoma of the nasopharynx represents a tumour with good prognosis but requires long term follow up in anticipation of local recurrence and progression to Multiple Myeloma.

\section{Keywords: extramedullary, plasmacytoma, nasopharynx}

Plasmacytomas are discrete tumours of solitary neoplastic plasma cells occuring in the bone (solitary plasmacytoma) or other soft tissues (extramedullary plasmacytoma). Extramedullary plasmacytoma is a rare localized tumour, first described by Schridde in 1905'. Three clinical manifestations exist: solitary, aggressive and disseminated. It is a rare neoplastic lesion that may occur in the head and neck region.' These lesions account for $4 \%$ of all nonepithelial tumors of the nasal cavity, paranasal sinuses and nasopharynx and they represent $0.4 \%$ of all head and neck malignancies. 'They have a slight predilection to men with peak incidence in patients aged 50 to 60 years. ${ }^{2}$ Extramedullary plasmacytoma can occur synchronously with multiple myeloma or can be antecedent to multiple myeloma years after, hence, the role of comprehensive multidisciplinary approach in long term management of such patients. The interrelationship between these different neoplastic plasma cell disorders was first described by Batsakis in 1983. ${ }^{3}$ The development of multiple myeloma has been observed in 8 to $36 \%$ of patients with extramedullary plasmacytoma. ${ }^{1,2}$ Whilst plasmacytomas tend to be discrete and solitary, multiple myeloma is diagnosed when there is diffuse infiltration of the marrow with neoplastic plasma cells. ${ }^{3}$ 


\section{CASE REPORT}

A 56-year-old gentleman with multiple co-morbidities presented to us in April 2003 with a 5-month history of right sided progressively worsening nasal blockage associated with intermittent self limiting epistaxis. This was associated with anosmia and occasional right sided temporal headaches. There was no history of loosening of teeth, facial swelling, diplopia or blurring of vision. Examination revealed a friable, dark red mass arising from the roof of the nasopharynx, completely occluding the right choana (Figure 1). Intraorally, there was inferior extension of the mass to the soft palate. There was no palpable cervical lymphadenopathy and cranial nerve examination was unremarkable. No other lesions were found in the head and neck region. He had a background history of hypertension, ischaemic heart disease and was an ex-chronic smoker.

Computed tomography showed a heterogeneously enhancing soft tissue density mass measuring $2.5 \mathrm{~cm}$ in its largest dimension, arising from the right post nasal space. No associated bony erosion or cervical lymphadenopathy was present. Biopsy of the mass revealed a nodular grayish tissue microscopically showing diffuse infiltration of the submucosal layer with plasma cells. The plasma cells were mature in appearance with eccentric nuclei and clock face chromatin pattern. Immunohistochemical studies revealed strong positivity towards lambda restriction and were negative for kappa, $L C A, B$ and T cell markers as well as cytokeratin. A histopathological diagnosis of extramedullary plasmacytoma of the nasopharynx was entertained. Urine and plasma electrophoresis were performed following this histological diagnosis, revealing no evidence of monoclonal band. Bone scan of the paranasal sinuses and skeletal surveys showed no lytic bony lesions suggestive of multiple myeloma. He underwent bone marrow and trephine biopsy to rule out marrow involvement. However, he developed cardiogenic shock secondary to unstable angina immediately following the procedure necessitating resuscitation and inotropic support. The bone marrow and trephine biopsy showed only occasional abnormal plasma cells of less than $5 \%$. He recovered from the cardiac event and was offered curative radiotherapy in view of his poor general medical condition. He subsequently underwent curative radiotherapy of $50 \mathrm{~Gy}$ for a total of 23 fractions to the nasopharynx.

He was well post radiotherapy with no further episodes of epistaxis or nasal obstruction. Total resolution of the mass was observed following radiotherapy with no subsequent evidence of local recurrence. Figure 4 shows an endoscopic view of the right choana 7 years post irradiation. This patient is receiving surveillance follow up from both the otorhinolaryngology and hematology teams. Up till his last visit at 7 years post irradiation, surveillance endoscopy, urine and serum paraprotein showed no significant evidence of local recurrence or development to multiple myeloma.
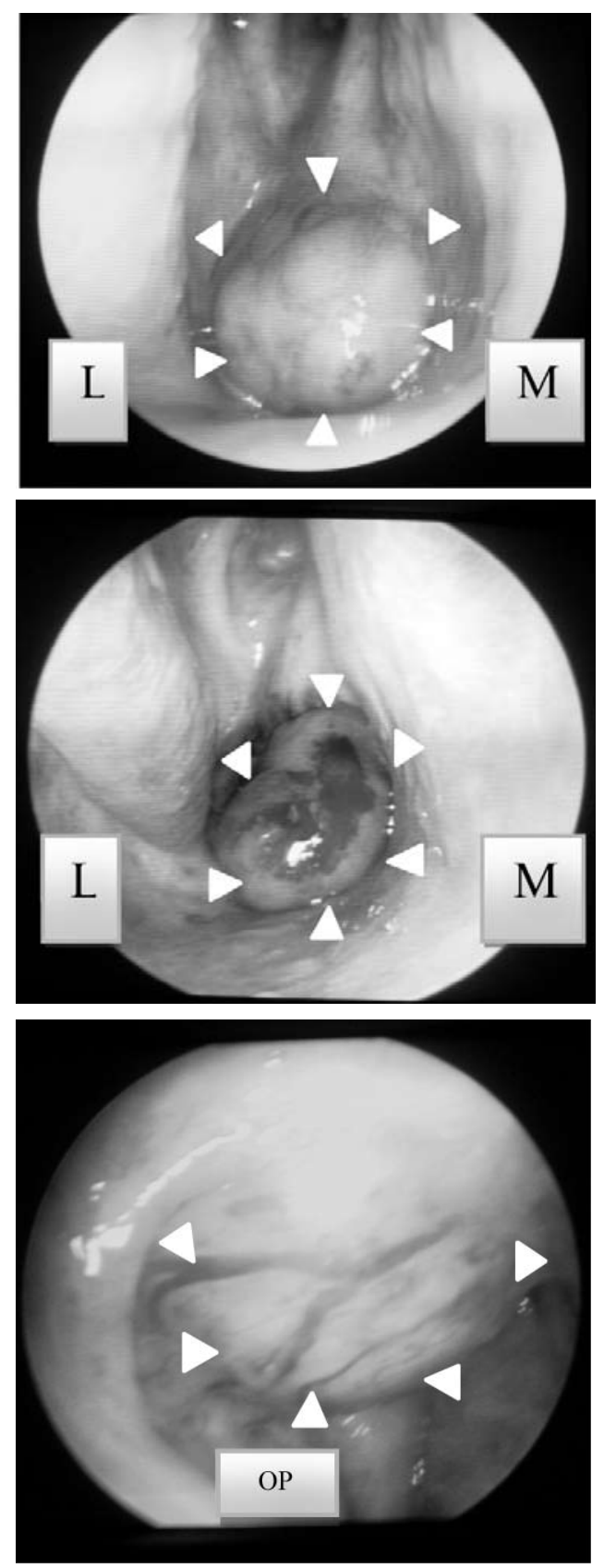

Figure 1: Endoscopic view ( $L$, lateral; $M$, medial) showing a friable mass completely occupying the right choana (arrowheads), extending inferiorly into the oropharynx (OP).

\section{DISCUSSION}

Plasma cell neoplastic disorders are important for otorhinolaryngologists to recognize since according to Wiltshaw in $1976,80 \%$ of extramedullary plasmacytomas occur in the head and neck and $10-20 \%$ of cases may present with multiple lesions. ${ }^{6}$ The etiology of extramedullary plasmacytoma is unknown. Proposed risk factors include chronic antigenic stimulation such as osteomyelitis, cholecystitis, rheumatoid arthritis and bacterial flora. Plasmacytoma 
formation has been demonstrated in laboratory mice treated with salmonella flagellar antigen and with bovine serum albumin. Genetic factors, radiation exposure, smoking and occupational exposures have also been implicated in the myeloma literature as possible etiologic agents. ${ }^{37,8}$

Wax, et al. in 1993 reviewed similar cases and found that 75\% of extramedullary plasmacytomas occurred in the sinonasal or nasopharyngeal area, $12 \%$ in the oropharynx, $8 \%$ in the larynx, and other sites in the head and neck including the tongue, minor salivary glands, thyroid, parotid, orbit and temporal bone..$^{10}$ Outside of the head and neck, extramedullary plasmacytoma has been reported in the pleura, mediastinum, spermatic cord, ovary, intestines, kidney, pancreas, breast, and skin. ${ }^{10}$ Most of the symptoms related to extramedullary plasmacytoma can be related to their specific location in the head and neck. Cervical lymph node metastasis is reported to occur in $12-26 \%$ of cases at initial presentation.

Biopsy of the lesion is the first step in confirming the diagnosis. Deep biopsies must be taken since the tumor is submucosal and the mucosa may be thickened from an inflammatory reaction. ' Histological subtypes of plasmacytoma including plasmacytic, plasmablastic and anaplastic subtypes have been described. However, these subtypes are neither indicative of prognosis or increased risk of recurrence. ${ }^{1,3}$ Local amyloid deposits have been found in 11-38\% of cases but systemic amyloidosis is very rare. Similar to multiple myeloma and other B cell neoplasms, a monoclonal staining pattern demonstrating either one heavy chain class, one light chain type or both can be demonstrated from immunohistochemical techniques, $3,7,8$ With new advances in immunophenotyping, CD 138 has been recognized as a marker for neoplastic plasma cells. ${ }^{17}$ However, it was not available in our centre at the point of diagnosis. Our immunohistochemical staining showed strong positivity to lambda restriction.

In a 1988 review of plasma cell disorders, Abemayor, et al. recommend a complete blood count with white blood cell count and platelet count, bone marrow biopsy, serum biochemistry including calcium, blood urea nitrogen, creatinine, uric acid, serum protein, serum and urine electophoresis, and a skeletal survey to rule out multiple myeloma. 'Galieni et al. suggested certain diagnostic criteria for solitary extramedullary plasmocytoma. They include biopsy of tissue showing monoclonal plasma cell histology, bone marrow plasma cell infiltration showing less than $5 \%$ of all nucleated cells, absence of any osteolytic bone lesion, absence of hypercalcemia or renal failure and low levels of paraprotein concentration if present. ${ }^{12}$ Our case satisfied all the criteria described above. High levels of paraprotein in the serum or urine should raise the clinician's suspicion of a disseminated process, since paraprotein levels correlate directly with tumor burden.

A review of therapeutic approaches to extramedullary plasmacytoma of the nasopharynx in published reports in the past ten years is discussed. Although similar cases have been described as early as 40 years ago, treatment has remained controversial. They include
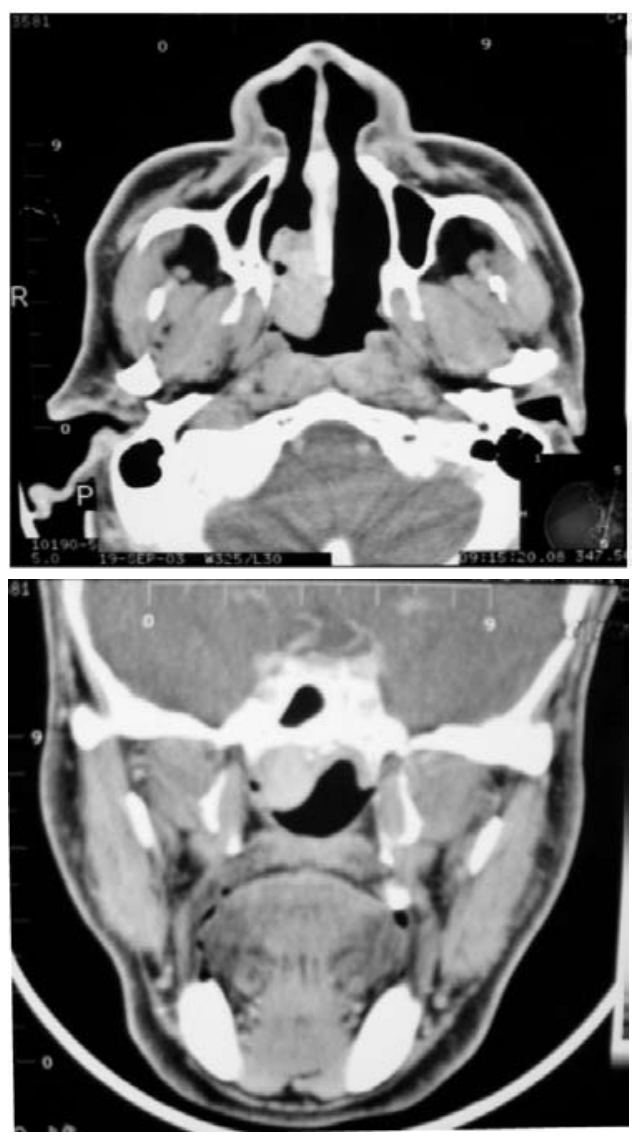

Figure 2: Computed Tomography, axial and coronal sections, showing mass arising from the right postnasal space extending inferiorly to the oropharynx without any bony involvement.

curative radiotherapy, curative surgical resection, salvage surgery and chemotherapy. ${ }^{13}$ While it is agreed that extramedullary plasmacytomas are radiosensistive, there is no consensus in the literature about the ideal dose of radiation therapy. Residual tumor is observed in $20 \%$ of patients after radiotherapy, so additional courses are sometimes necessary. ${ }^{14}$ Alexiou et al. suggested that surgery alone gave the best results for extramedullary plasmacytoma of the upper aero-digestive tract when resectability is good. However, if complete surgical resection was not possible or doubtful, and/or lymph nodes were affected, then combined therapy (surgery and radiation) was recommended. ${ }^{15}$ There are very few reports of local recurrence of plasmacytomas treated primarily by surgery.${ }^{16}$ Most authors recommended salvage surgery if there is local failure following additional courses of radiotherapy. Salvage surgery following radiotherapy often yields more amyloid component rather than tumour tissue, suggesting its radiosensitive properties. ${ }^{11}$ Rubin et al. in 1990 reviewed 14 series and 219 cases of extramedullary plasmacytoma of the head and neck in the literature. They found that $55 \%$ of patients treated with radiotherapy alone and $54 \%$ of patients treated with surgery alone had no evidence of 

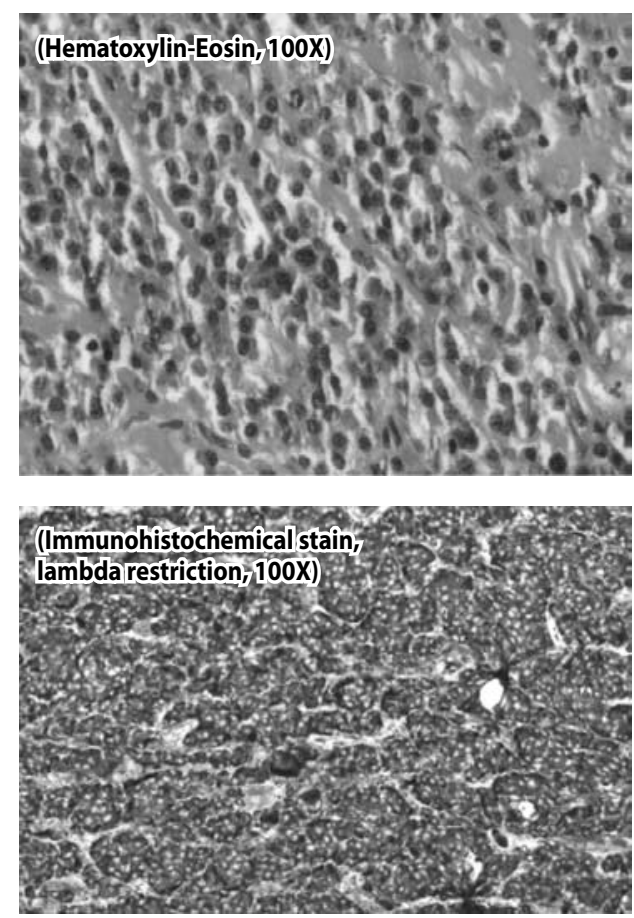

Figure 3: Diffuse infiltration of the submucosal layer with plasma cells seen under high power view (Hematoxylin - Eosin staining, magnification 100X). The latter showing immunohistochemical staining positive towards lambda restriction (magnification 100X)
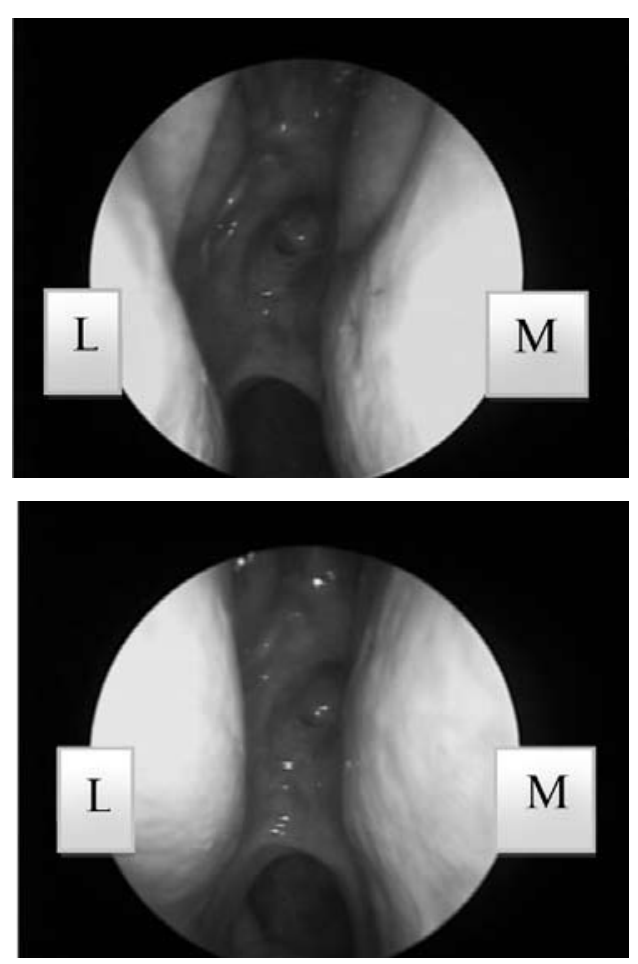

Figure 4: Endoscopic view of the right choana, 7 years post irradiation (2010) showing no evidence of local recurrence. L: lateral wall of the nasal cavity M: medial wall of the nasal cavity recurrence post treatment. Mortality and recurrence were also noted to not be significantly different between the two groups. Chemotherapy and bone marrow transplantation is reserved for disseminated disease or progression to multiple myeloma. ${ }^{9}$

Factors associated with poor prognosis in the literature include the presence of bone destruction, large primary tumor, recurrence, and tumors located in the sphenoid, maxillary sinus, orbit, and larynx. ${ }^{6}$ Histologic appearance and lymph node involvement are not reported to be of any prognostic significance. Because extramedullary plasmacytoma can recur as disseminated multiple myeloma long term follow-up likewise in our patient is important. Cases of recurrence have been reported 28 and 36 years after initial treatment in the literature. ${ }^{6}$ While most diseases are considered treated following five years of being recurrence free, such is not the case in plasmacytoma. Therefore, we plan for lifetime follow up in this patient, diligently anticipating any possible recurrence or progression to multiple myeloma.

Extramedullary plasmacytoma of the nasopharynx represents a tumour with good prognosis but requires long term follow up in anticipation of local recurrence and progression to multiple myeloma.

\section{REFERENCES}

1. Miller FR, Lavertu P, Wanamaker JR, Bonafede J, Wood BG. Plasmacytomas of the head and neck. Otolaryngol Head Neck Surg 1998 Dec; 119(6):614-18.

2. Paris J, Dessi P, Moulin G, Chrestian MA, Braccini F, Zanaret M. Extramedullary plasmacytoma of the nasal cavity: A case report. Rev Laryngol Otol Rhinol 1999; 120(5): 343-5.

3. Batsakis JG. Plasma cell tumors of head and neck. Pathology consultation. Ann. Otol. Rhinol. Laryngol. 1983 May-Jun;92:311-313.

4. Knowling MA, Harwood AR, Bergsagel DE. Comparison of extramedullary plasmacytomas with solitary and multiple plasma cell tumors of bone. J Clin Oncol 1983 Apr; 1(4):255-62.

5. Holland J, Trenker DA, Wasserman TH, Fineberg B. Plasmacytoma. Treatment results and conversion to myeloma. Cancer 1992 Mar 15; 69 (6): 1513-17.

6. Wiltshaw $E$. The natural history of extramedullary plasmacytoma and its relation to solitary myeloma of bone and myelomatosis. Medicine (Baltimore) 1976 May; 55(3) 217-38.

7. Abemayor E, Canalis RF, Greenberg P, Wortham DG, Rowland JP, Sun NC. Plasma cell tumors of the head and neck. J Otolaryngol. 1988 Dec; 17(17):376-381.

8. Batsakis JG, Fries GT, Goldman RT, Karlseberg RC. Upper respiratory tract plasmacytoma. Arch Otolaryngol. 1964 Jun; 79:613-618.

9. Rubin J, Johnson JT, Killeen R, Barnes L. Extramedullary plasmacytoma of the thyroid associated with a serum monoclonal gammopathy. Arch Otolaryngol Head Neck Surg. 1990;116(7):855-859.

10.Wax MK, Yun KJ, Omar RA. Extramedullary plasamacytomas of the head and neck. Otolaryngol. Head Neck Surg. 1993 Nov;109(5):877-888.

11. Hidaka H, Ikeda K, Oshima T, Ohtani H, Suzuki H, Takasaka T. A case of extramedullary plasmacytoma arising from the nasal septum. J Laryngol Otol. 2000 Jan; 114(1):53-5.

12.Galieni P, Cavo M, Pulsoni A, Awisati G, Bigazzi C, Neri S, et al: Clinical outcome of extramedullary plasmacytoma. Haematologica $2000 \mathrm{Jan} ; 85(1): 47-51$.

13. Yavas O, Altundag K, Sungur A. Extramedullary plasmacytoma of nasopharynx and larynx: Synchronous presentation. Am J Hematol 2004 Apr; 75 (4): 264-5.

14.Tesei F, Caliceti U, Sorrenti G, Canciullo A, Sabbatini E, Pileri S, et al. Extramedullary plasmocytoma (EMP) of the head and neck: A series of 22 cases;. Acta Otorhinolaryngol Ital. 1995 Dec; 15 (6): 437-42.

15.Alexiou C, Kau RJ, Dietzfelbinger H, Kremer M, Spiess JC, Schratzenstaller, et al. Extramedullary plasmacytoma; tumour occurrence and therapeutic concepts. Cancer 2000 Jan 1; 88(1): 240-242.

16. Soesan M, Paccagnella A, Chiarion-Sileni V, Salvagno L, Fornasiero A, Sotti G, et al. Extramedullary plasmacytoma: Clinical behaviour and response to treatment. Ann Oncol. 1992 Jan; 3(1) :51-57.

17.O'Connell FP, Pinkus JL, Pinkus GS.). CD138 (syndecan-1), a plasma cell marke immunohistochemical profile in hematopoietic and nonhematopoietic neoplasms. Am. J. Clin. Pathol. 2004 Feb; 121 (2): 254-63. 